\title{
Existence and multiplicity of solutions for nonlocal $p(x)$-Laplacian equations with nonlinear Neumann boundary conditions
}

Erlin Guo ${ }^{*}$ and Peihao Zhao

* Correspondence: guoerlin@lzu.
edu.cn
School of Mathematics and
Statistics, Lanzhou University,
Lanzhou 730000, P. R. China

* Correspondence: guoerlin@lzu.

School of Mathematics and Lanzhou 730000, P. R. China

\section{Abstract}

In this article, we study the nonlocal $p(x)$-Laplacian problem of the following form

$$
\left\{\begin{array}{c}
a\left(\int_{\Omega} \frac{1}{p(x)}\left(|\nabla u|^{p(x)}+|u|^{p(x)}\right) d x\right)\left(-\operatorname{div}\left(|\nabla u|^{p(x)-2} \nabla u\right)+|u|^{p(x)-2} u\right) \\
=b\left(\int_{\Omega} F(x, u) \mathrm{d} x\right) f(x, u) \text { in } \Omega \\
a\left(\int_{\Omega} \frac{1}{p(x)}\left(|\nabla u|^{p(x)}+|u|^{p(x)}\right) d x\right)|\nabla u|^{p(x)-2} \frac{\partial u}{\partial v}=g(x, u) \text { on } \partial \Omega,
\end{array}\right.
$$

where $\Omega$ is a smooth bounded domain and $v$ is the outward normal vector on the boundary $\partial \Omega$, and $F(x, u)=\int f(x, t) d t$. By using the variational method and the theory of the variable exporeht Sobolev space, under appropriate assumptions on $f$, $g, a$ and $b$, we obtain some results on existence and multiplicity of solutions of the problem.

Mathematics Subject Classification (2000): 35B38; 35D05; 35J20.

Keywords: critical points, $p(x)$-Laplacian, nonlocal problem, variable exponent Sobolev spaces, nonlinear Neumann boundary conditions

\section{Introduction}

In this article, we consider the following problem

$$
(P)\left\{\begin{array}{c}
a\left(\int_{\Omega} \frac{1}{p(x)}\left(|\nabla u|^{p(x)}+|u|^{p(x)}\right) d x\right)\left(-\operatorname{div}\left(|\nabla u|^{p(x)-2} \nabla u\right)+|u|^{p(x)-2} u\right) \\
=b\left(\int_{\Omega} F(x, u) \mathrm{d} x\right) f(x, u) \text { in } \Omega \\
a\left(\int_{\Omega} \frac{1}{p(x)}\left(|\nabla u|^{p(x)}+|u|^{p(x)}\right) d x\right)|\nabla u|^{p(x)-2} \frac{\partial u}{\partial v}=g(x, u) \text { on } \partial \Omega,
\end{array}\right.
$$

where $\Omega$ is a smooth bounded domain in $R^{N}, p \in C(\bar{\Omega})$ with $1<p^{-}:=\inf _{\Omega} p(x) \leq p$ $(x) \leq p^{+}:=\sup _{\Omega} p(x)<N, a(t)$ is a continuous real-valued function, $f: \Omega \times R \rightarrow R, g$ : $\partial \Omega \times R \rightarrow R$ satisfy the Caratheodory condition, and $F(x, u)=\int_{0}^{u} f(x, t) d t$. Since the equation contains an integral related to the unknown $u$ over $\Omega$, it is no longer an identity pointwise, and therefore is often called nonlocal problem. 
Kirchhoff [1] has investigated an equation

$$
\rho \frac{\partial^{2} u}{\partial t^{2}}-\left(\frac{P_{0}}{h}+\frac{E}{2 L} \int_{0}^{L}\left|\frac{\partial u}{\partial x}\right|^{2} d x\right) \frac{\partial^{2} u}{\partial x^{2}}=0,
$$

which is called the Kirchhoff equation. Various equations of Kirchhoff type have been studied by many authors, especially after the work of Lions [2], where a functional analysis framework for the problem was proposed; see e.g. [3-6] for some interesting results and further references. In the following, a key work on nonlocal elliptic problems is the article by Chipot and Rodrigues [7]. They studied nonlocal boundary value problems and unilateral problems with several applications. And now the study of nonlocal elliptic problem has already been extended to the case involving the $p$ Laplacian; see e.g. [8,9]. Recently, Autuori, Pucci and Salvatori [10] have investigated the Kirchhoff type equation involving the $\mathrm{p}(\mathrm{x})$-Laplacian of the form

$$
u_{t t}-M\left(\int_{\Omega} \frac{1}{p(x)}|\nabla u|^{p(x)} d x\right) \Delta_{p(x)} u+Q\left(t, x, u, u_{t}\right)+f(x, u)=0 .
$$

The study of the stationary version of Kirchhoff type problems has received considerable attention in recent years; see e.g. [5,11-16].

The operator $\Delta_{p(x)} \mathrm{u}=\operatorname{div}\left(|\nabla u|^{p(x)-2} \nabla u\right)$ is called $p(x)$-Laplacian, which becomes $p$ Laplacian when $p(x) \equiv p$ (a constant). The $p(x)$-Laplacian possesses more complicated nonlinearities than $p$-Laplacian. The study of various mathematical problems with variable exponent are interesting in applications and raise many difficult mathematical problems. We refer the readers to [17-23] for the study of $p(x)$-Laplacian equations and the corresponding variational problems.

Corrêa and Figueiredo [13] presented several sufficient conditions for the existence of positive solutions to a class of nonlocal boundary value problems of the $p$-Kirchhoff type equation. Fan and Zhang [20] studied $p(x)$-Laplacian equation with the nonlinearity $f$ satisfying Ambrosetti-Rabinowitz condition. The $\mathrm{p}(\mathrm{x})$-Kirchhoff type equations with Dirichlet boundary value problems have been studied by Dai and Hao [24], and much weaker conditions have been given by Fan [25]. The elliptic problems with nonlinear boundary conditions have attracted expensive interest in recent years, for example, for the Laplacian with nonlinear boundary conditions see [26-30], for elliptic systems with nonlinear boundary conditions see [31,32], for the $p$-Laplacian with nonlinear boundary conditions of different type see [33-37], and for the $p(x)$-Laplacian with nonlinear boundary conditions see [38-40]. Motivated by above, we focus the case of nonlocal $p(x)$-Laplacian problems with nonlinear Neumann boundary conditions. This is a new topics even when $p(x) \equiv p$ is a constant.

This rest of the article is organized as follows. In Section 2, we present some necessary preliminary knowledge on variable exponent Sobolev spaces. In Section 3, we consider the case where the energy functional associated with problem $(P)$ is coercive. And in Section 4, we consider the case where the energy functional possesses the Mountain Pass geometry.

\section{Preliminaries}

In order to discuss problem $(P)$, we need some theories on variable exponent Sobolev space $W^{1, p(x)}(\Omega)$. For ease of exposition we state some basic properties of space $W^{1, p(x)}$ $(\Omega)$ (for details, see $[22,41,42]$ ). 
Let $\Omega$ be a bounded domain of $R^{N}$, denote

$$
\begin{aligned}
& C_{+}(\bar{\Omega})=\{p \mid p \in C(\bar{\Omega}), p(x)>1, \forall x \in \bar{\Omega}\}, \\
& p^{+}=\max _{x \in \bar{\Omega}} p(x), p^{-}=\min _{x \in \bar{\Omega}} p(x), \forall p \in C(\bar{\Omega}), \\
& L^{p(x)}(\Omega)=\left\{u \mid u \text { is a measurable real - valued function on } \Omega, \int_{\Omega}|u|^{p(x)} \mathrm{d} x<\infty\right\},
\end{aligned}
$$

we can introduce the norm on $L^{p(x)}(\Omega)$ by

$$
|u|_{p(x)}=\inf \left\{\lambda>0: \int_{\Omega}\left|\frac{u(x)}{\lambda}\right|^{p(x)} \mathrm{d} x \leq 1\right\}
$$

and $\left(L^{p(x)}(\Omega),|\cdot|_{p(x)}\right)$ becomes a Banach space, we call it the variable exponent Lebesgue space.

The space $W^{1, p(x)}(\Omega)$ is defined by

$$
W^{1, p(x)}(\Omega)=\left\{u \in L^{p(x)}(\Omega) \| \nabla u \mid \in L^{p(x)}(\Omega)\right\},
$$

and it can be equipped with the norm

$$
\|u\|=|u|_{p(x)}+|\nabla u|_{p(x)},
$$

where $|\nabla u|_{p(x)}=\|\left.\nabla u\right|_{p(x)}$; and we denote by $W_{0}^{1, p(x)}(\Omega)$ the closure of $C_{0}^{\infty}(\Omega)$ in $W^{1}$, $p(x)(\Omega), p^{*}=\frac{N p(x)}{N-p(x)}, p_{*}=\frac{(N-1) p(x)}{N-p(x)}$, when $p(x)<N$, and $p^{*}=p_{*}=\infty$, when $p(x)>N$.

Proposition $2.1[22,41]$. (1) If $p \in C_{+}(\bar{\Omega})$, the space $\left(L^{p(x)}(\Omega),|\cdot|_{p(x)}\right)$ is a separable, uniform convex Banach space, and its dual space is $L^{q(x)}(\Omega)$, where $1 / q(x)+1 / p(x)=$ 1. For any $u \in L^{p(x)}(\Omega)$ and $v \in L^{q(x)}(\Omega)$, we have

$$
\left|\int_{\Omega} u v \mathrm{~d} x\right| \leq\left(\frac{1}{p-}+\frac{1}{q-}\right)|u|_{p(x)}|v|_{q(x)}
$$

(2) If $p_{1}, p_{2} \in C_{+}(\bar{\Omega}), p_{1}(x) \leq p_{2}(x)$, for any $x \in \Omega$, then $L^{p_{2}(x)}(\Omega) \hookrightarrow L^{p_{1}(x)}(\Omega)$, and the imbedding is continuous.

Proposition 2.2 [22]. If $f: \Omega \times R \rightarrow R$ is a Caratheodory function and satisfies

$$
|f(x, s)| \leq d(x)+e|s|^{\frac{p_{1}(x)}{p_{2}(x)}}, \quad \text { for any } x \in \Omega, s \in R,
$$

where $p_{1}, p_{2} \in C_{+}(\bar{\Omega}), d \in L^{p_{2}(x)}(\Omega), d(x) \geq 0$ and $e \geq 0$ is a constant, then the superposition operator from $L^{p_{1}(x)}(\Omega)$ to $L^{p_{2}(x)}(\Omega)$ defined by $\left(N_{f}(u)\right)(x)=f(x, u(x))$ is a continuous and bounded operator.

Proposition 2.3 [22]. If we denote

$$
\rho(u)=\int_{\Omega}|u|^{p(x)} \mathrm{d} x, \quad \forall u \in L^{p(x)}(\Omega),
$$

then for $u, u_{n} \in L^{p(x)}(\Omega)$

(1) $|u(x)|_{p(x)}<1(=1 ;>1) \Leftrightarrow \rho(u)<1(=1 ;>1)$;

$$
\begin{aligned}
& |u(x)|_{p(x)}>1 \Rightarrow|u|_{p(x)}^{p^{-}} \leq \rho(u) \leq|u|_{p(x)^{+}}^{p^{+}} ; \\
& |u(x)|_{p(x)}<1 \Rightarrow|u|_{p(x)}^{p^{-}} \geq \rho(u) \geq|u|_{p(x)^{\prime}}^{p^{+}} ; \\
& \text {(3) } \\
& \left|u_{n}(x)\right|_{p(x)} \rightarrow 0 \Leftrightarrow \rho\left(u_{n}\right) \rightarrow 0 \text { as } n \rightarrow \infty \text {; } \\
& \left|u_{n}(x)\right|_{p(x)} \rightarrow \infty \Leftrightarrow \rho\left(u_{n}\right) \rightarrow \infty \text { as } n \rightarrow \infty \text {. }
\end{aligned}
$$


Proposition 2.4 [22]. If $u, u_{n} \in L^{p(x)}(\Omega), n=1,2, \ldots$, then the following statements are equivalent to each other

(1) $\lim _{k \rightarrow \infty}\left|u_{k}-u\right|_{p(x)}=0$;

(2) $\lim _{k \rightarrow \infty} \rho\left|u_{k}-u\right|=0$;

(3) $u_{k} \rightarrow u$ in measure in $\Omega$ and $\lim _{k \rightarrow \infty} \rho\left(u_{k}\right)=\rho(u)$.

Proposition 2.5 [22]. (1) If $p \in C_{+}(\bar{\Omega})$, then $W_{0}^{1, p(x)}(\Omega)$ and $W^{1, p(x)}(\Omega)$ are separable reflexive Banach spaces;

(2) if $q \in C_{+}(\bar{\Omega})$ and $q(x)<p^{*}(x)$ for any $x \in \bar{\Omega}$, then the imbedding from $W^{1, p(x)}(\Omega)$ to $L^{q(x)}(\Omega)$ is compact and continuous;

(3) if $q \in C_{+}(\bar{\Omega})$ and $q(x)<p_{*}(x)$ for any $x \in \bar{\Omega}$, then the trace imbedding from $W^{1, p}$

${ }^{(x)}(\Omega)$ to $L^{q(x)}(\partial \Omega)$ is compact and continuous;

(4) (Poincare inequality) There is a constant $C>0$, such that

$$
|u|_{p(x)} \leq C|\nabla u|_{p(x)} \quad \forall u \in W_{0}^{1, p(x)}(\Omega) .
$$

So, $|\nabla u|_{p(x)}$ is a norm equivalent to the norm $\|u\|$ in the space $W_{0}^{1, p(x)}(\Omega)$.

\section{Coercive functionals}

In this and the next sections we consider the nonlocal $p(x)$-Laplacian-Neumann problem $(P)$, where $a$ and $b$ are two real functions satisfying the following conditions

$\left(\mathrm{a}_{1}\right) a:(0,+\infty) \rightarrow(0,+\infty)$ is continuous and $\mathrm{a} \in L^{1}(0, t)$ for any $t>0$.

$\left(\mathrm{b}_{1}\right) b: R \rightarrow R$ is continuous.

Notice that the function $a$ satisfies $\left(\mathrm{a}_{1}\right)$ may be singular at $t=0$. And $f, g$ satisfying

$\left(\mathrm{f}_{1}\right) f: \Omega \times R \rightarrow R$ satisfies the Caratheodory condition and there exist two constants $C_{1} \geq 0, C_{2} \geq 0$ such that

$$
|f(x, t)| \leq C_{1}+C_{2}|t|^{q_{1}(x)-1}, \forall(x, t) \in \Omega \times R,
$$

where $q_{1} \in C_{+}(\bar{\Omega})$ and $q_{1}(x)<p^{*}(x), \forall x \in \bar{\Omega}$.

( $\left.\mathrm{g}_{1}\right) g: \partial \Omega \times R \rightarrow R$ satisfies the Caratheodory condition and there exist two constants $C_{1}^{\prime} \geq 0, C_{2}^{\prime} \geq 0$ such that

$$
|g(x, t)| \leq C_{1}^{\prime}+C_{2}^{\prime}|t|^{q_{2}(x)-1}, \forall(x, t) \in \partial \Omega \times R,
$$

where $q_{2} \in C_{+}(\partial \Omega)$ and $q_{2}(x)<p^{*}(x), \forall x \in \partial \Omega$. For simplicity we write $\mathrm{X}=W^{1, p(x)}$ $(\Omega)$, denote by $C$ the general positive constant (the exact value may change from line to line).

Define

$$
\begin{aligned}
& \widehat{a}(t)=\int_{0}^{t} a(s) d s, \widehat{b}(t)=\int_{0}^{t} b(s) d s, \forall t \in R, \\
& I_{1}(u)=\int_{\Omega} \frac{1}{p(x)}\left(|\nabla u|^{p(x)}+|u|^{p(x)}\right) d x, I_{2}(u)=\int_{\Omega} F(x, u) d x, \forall u \in X, \\
& J(u)=\widehat{a}\left(I_{1}(u)\right)=\widehat{a}\left(\int_{\Omega} \frac{1}{p(x)}\left(|\nabla u|^{p(x)}+|u|^{p(x)}\right) d x\right), \\
& \Phi(u)=\widehat{b}\left(I_{2}(u)\right)=\widehat{b}\left(\int_{\Omega} F(x, u) \mathrm{d} x\right) \text { and } \Psi(u)=\int_{\partial \Omega} G(x, u) \mathrm{d} \sigma, \forall u \in X, \\
& E(u)=J(u)-\Phi(u)-\Psi(u), \forall u \in X, \\
& \text { where } F(x, u)=\int_{0}^{u} f(x, t) d t, G(x, u)=\int_{0}^{u} g(x, t) \mathrm{d} t .
\end{aligned}
$$


Lemma 3.1. Let $\left(f_{1}\right),\left(g_{1}\right)\left(a_{1}\right)$ and $\left(b_{1}\right)$ hold. Then the following statements hold true:

(1) $\widehat{a} \in C^{0}([0, \infty)) \cap C^{1}((0, \infty)), \widehat{a}(0)=0, \widehat{a}^{\prime}(t)=a(t)>0 ; \widehat{b} \in C^{1}(R), \widehat{b}(0)=0$.

(2) $J, \Phi, \Psi$ and $E \in C^{0}(X), J(0)=\Phi(0)=\Psi(0)=E(0)=0$. Furthermore $J \in C^{1}(X$ $\backslash\{0\}), \Phi, \Psi \in C^{1}(X), E \in C^{1}(X \backslash\{0\})$. And for every $u \in X \backslash\{0\}, v \in X$, we have

$$
\begin{aligned}
E^{\prime}(u) v= & a\left(\int_{\Omega} \frac{1}{p(x)}\left(|\nabla u|^{p(x)}+|u|^{p(x)}\right) d x\right) \int_{\Omega}\left(|\nabla u|^{p(x)-2} \nabla u \nabla v+|u|^{p(x)-2} u v\right) \mathrm{d} x \\
& -b\left(\int_{\Omega} F(x, u) \mathrm{d} x\right) \int_{\Omega} f(x, u) v \mathrm{~d} x-\int_{\partial \Omega} g(x, u) v \mathrm{~d} \sigma .
\end{aligned}
$$

Thus $u \in X \backslash\{0\}$ is a (weak) solution of $(P)$ if and only if $u$ is a critical point of $E$.

(3) The functional $J: X \rightarrow R$ is sequentially weakly lower semi-continuous, $\Phi, \Psi: X$ $\rightarrow R$ are sequentially weakly continuous, and thus $E$ is sequentially weakly lower semicontinuous.

(4) The mappings $\Phi^{\prime}$ and $\Psi^{\prime}$ are sequentially weakly-strongly continuous, namely, $u_{n}$ $\rightarrow u$ in $X$ implies $\Phi^{\prime}\left(u_{n}\right) \rightarrow \Phi^{\prime}(u)$ in $X^{*}$. For any open set D $\subset X \backslash\{0\}$ with $\bar{D} \subset X \backslash\{0\}$, The mappings $\rho$ and $E^{\prime}: \bar{D} \rightarrow X^{*}$ are bounded, and are of type $\left(S_{+}\right)$, namely,

$$
u_{n} \rightarrow u \text { and } \varlimsup_{n \rightarrow \infty} J^{\prime}\left(u_{n}\right)\left(u_{n}-u\right) \leq 0 \text {, implies } u_{n} \rightarrow u \text {. }
$$

Definition 3.1. Let $c \in \mathrm{R}$, a $C^{1}$-functional $E: X \rightarrow R$ satisfies (P.S) $)_{c}$ condition if and only if every sequence $\left\{u_{j}\right\}$ in $X$ such that $\lim _{j} E\left(u_{j}\right)=c$, and $\lim _{j} E^{\prime}\left(u_{j}\right)=0$ in $X^{*}$ has a convergent subsequence.

Lemma 3.2. Let $\left(f_{1}\right),\left(g_{1}\right),\left(a_{1}\right),\left(b_{1}\right)$ hold. Then for any $c \neq 0$, every bounded (P. S $)_{c}$ sequence for $E$, i.e., a bounded sequence $\left\{u_{n}\right\} \subset X \backslash\{0\}$ such that $E\left(u_{n}\right) \rightarrow c$ and $E\left(u_{n}\right)$ $\rightarrow 0$, has a strongly convergent subsequence.

The proof of these two lemmas can be obtained easily from [25,40], we omitted them here.

Theorem 3.1. Let $\left(f_{1}\right),\left(g_{1}\right),\left(a_{1}\right),\left(b_{1}\right)$ and the following conditions hold true:

$\left(\mathrm{a}_{2}\right)$ There are positive constants $\alpha_{1}, M$, and $C$ such that $\widehat{a}(t) \geq C t^{\alpha_{1}}$ for $t \geq M$.

$\left(\mathrm{b}_{2}\right)$ There are positive constants $\beta_{1}$ and $C$ such that $|\widehat{b}(t)| \leq C+C|t|^{\beta_{1}}$ for $t \in R$.

$\left(\mathrm{H}_{1}\right) \beta_{1} q_{1+}<\alpha_{1} p_{-}, q_{2+}<\alpha_{1} p_{-}$.

Then the functional $E$ is coercive and attains its infimum in $X$ at some $u_{0} \in X$. Therefore, $u_{0}$ is a solution of $(P)$ if $E$ is differentiable at $u_{0}$.

Proof. For $\|u\|$ large enough, by $\left(\mathrm{f}_{1}\right),\left(\mathrm{g}_{1}\right),\left(\mathrm{a}_{2}\right),\left(\mathrm{b}_{2}\right)$ and $\left(\mathrm{H}_{1}\right)$, we have that

$$
\begin{aligned}
& J(u)=\widehat{a}\left(I_{1}(u)\right)=\widehat{a}\left(\int_{\Omega} \frac{1}{p(x)}\left(|\nabla u|^{p(x)}+|u|^{p(x)}\right) d x\right) \\
& \geq \widehat{a}\left(C_{1}\|u\|^{p-}\right) \geq C_{2}\|u\|^{\alpha_{1} p-}, \\
& \left|\int_{\Omega} F(x, u) \mathrm{d} x\right| \leq C_{3}\|u\|^{q_{1}+}, \\
& \Phi(u)=\widehat{b}\left(I_{2}(u)\right)=\widehat{b}\left(\int_{\Omega} F(x, u) \mathrm{d} x\right) \leq C_{4}\|u\|^{\beta_{1} q_{1}+}+\widetilde{C_{4}}, \\
& \Psi(u)=\left|\int_{\partial \Omega} G(x, u) \mathrm{d} \sigma\right| \leq C_{5}|| u \|^{q_{2+}}+\widetilde{C_{5}}, \\
& E(u)=J(u)-\Phi(u)-\Psi(u) \geq C_{2}\|u\|^{\alpha_{1} p-}-C_{4}\|u\|^{\beta_{1} q_{1+}}-C_{5}\|u\|^{q_{2+}}-+\widetilde{C_{6}},
\end{aligned}
$$


and hence $E$ is coercive. Since $E$ is sequentially weakly lower semi-continuous and $X$ is reflexive, $E$ attains its infimum in $X$ at some $u_{0} \in X$. In this case $E$ is differentiable at $u_{0}$, then $u_{0}$ is a solution of $(P)$.

Theorem 3.2. Let $\left(f_{1}\right),\left(g_{1}\right),\left(a_{1}\right),\left(b_{1}\right),\left(a_{2}\right),\left(b_{2}\right),\left(H_{1}\right)$ and the following conditions hold true:

$\left(\mathrm{a}_{3}\right)$ There is a positive constant $\alpha_{2}$ such that $\limsup _{t \rightarrow 0+} \frac{\hat{a}(t)}{t^{\alpha_{2}}}<+\infty$.

$\left(b_{3}\right)$ There is a positive constant $\beta_{2}$ such that $\liminf _{t \rightarrow 0} \frac{\widehat{b}(t)}{|t|^{\beta_{2}}}>0$.

$\left(\mathrm{f}_{2}\right)$ There exist an open subset $\Omega_{0}$ of $\Omega$ and $r_{1}>0$ such that $\lim _{t \rightarrow 0} \inf _{t \rightarrow t^{2}} \frac{F(x, t)}{\mid t r_{2}}>0$ uniformly for $x \in \Omega_{0}$.

$\left(\mathrm{g}_{2}\right)$ There exists $r_{2}>0$ such that $\liminf _{t \rightarrow 0} \frac{G(x, t)}{|t|^{2}}>0$ uniformly for $x \in \partial \Omega$.

$\left(\mathrm{H}_{2}\right) \beta_{2} r_{1}<\alpha_{2} p_{-}, r_{2}<\alpha_{2} p_{\text {. }}$.

Then $(P)$ has at least one nontrivial solution which is a global minimizer of the energy functional $E$.

Proof. From Theorem 3.1 we know that $E$ has a global minimizer $u_{0}$. It is clear that $\widehat{b}(0)=0, \widehat{b}(0)=0, F(x, 0)$ and consequently E $(0)=0$. Take $w \in C_{0}^{\infty}\left(\Omega_{0}\right) \backslash\{0\}$. Then, by $\left(f_{2}\right),\left(g_{2}\right)\left(a_{3}\right),\left(b_{3}\right)$ and $\left(H_{2}\right)$, for sufficiently small $\lambda>0$ we have that

$$
\begin{aligned}
E(\lambda w)= & \widehat{a}\left(\int_{\Omega} \frac{\lambda^{p(x)}}{p(x)}\left(|\nabla w|^{p(x)}+|w|^{p(x)}\right) d x\right) \\
& -\widehat{b}\left(\int_{\Omega} F(x, \lambda w) \mathrm{d} x\right)-\int_{\partial \Omega} G(x, \lambda w) \mathrm{d} \sigma \\
\leq & C_{1}\left(\int_{\Omega} \frac{\lambda^{p(x)}}{p(x)}\left(|\nabla w|^{p(x)}+|w|^{p(x)}\right) d x\right)^{\alpha_{2}} \\
& \left.-C_{2}\left(\int_{\Omega_{0}} F(x, \lambda w) \mathrm{d} x\right)\right)^{\beta_{2}}-C_{3} \int_{\partial \Omega}|\lambda w|^{r_{2}} \mathrm{~d} \sigma \\
\leq & C_{4} \lambda^{\alpha_{2} p-}-C_{5} \lambda^{\beta_{2} r_{1}}-C_{6} \lambda^{r_{2}}<0 .
\end{aligned}
$$

Hence $E\left(u_{0}\right)<0$ and $u_{0} \neq 0$.

By the genus theorem, similarly in the proof of Theorem 4.3 in [18], we have the following:

Theorem 3.3. Let the hypotheses of Theorem 3.2 hold, and let, in addition, $f$ and $g$ satisfy the following conditions:

$\left(\mathrm{f}_{3}\right) f(x,-t)=-f(x, t)$ for $x \in \Omega$ and $t \in R$.

$\left(\mathrm{g}_{3}\right) g(x,-t)=-g(x, t)$ for $x \in \partial \Omega$ and $t \in R$.

Then $(P)$ has a sequence of solutions $\left\{u_{n}\right\}$ such that $E\left(u_{n}\right)<0$.

Theorem 3.4. Let $\left(f_{1}\right),\left(g_{1}\right),\left(a_{1}\right),\left(b_{1}\right),\left(a_{2}\right),\left(b_{2}\right),\left(a_{3}\right),\left(b_{3}\right),\left(H_{1}\right),\left(H_{2}\right)$ and the following conditions hold true:

$\left(\mathrm{b}_{+}\right) b(t) \geq 0$ for $t \geq 0$.

$\left(\mathrm{f}_{+}\right) f(x, t) \geq 0$ for $x \in \Omega$ and $t \geq 0$.

$\left(\mathrm{g}_{+}\right) g(x, t) \geq 0$ for $x \in \partial \Omega$ and $t \geq 0$.

$\left(\mathrm{f}_{2}\right)_{+}$There exist an open subset $\Omega_{0}$ of $\Omega$ and $r_{1}>0$ such that $\liminf _{t \rightarrow 0+} \frac{F(x, t)}{t^{\prime} 1}>0$ uniformly for $x \in \Omega_{0}$.

$\left(\mathrm{g}_{2}\right)_{+}$There exists $r_{2}>0$ such that $\liminf _{t \rightarrow 0^{+}} \frac{G(x, t)}{t^{2} 2}>0$ uniformly for $x \in \partial \Omega$. 
Then $(P)$ has at least one nontrivial nonnegative solution with negative energy.

Proof. Define

$$
\begin{aligned}
& \tilde{f}(x, t)=\left\{\begin{array}{l}
f(x, t) \text { if } t \geq 0, \\
f(x, 0) \text { if } t<0,
\end{array} \quad \tilde{g}(x, t)=\left\{\begin{array}{l}
g(x, t) \text { if } t \geq 0, \\
g(x, 0) \text { if } t<0,
\end{array}\right.\right. \\
& \tilde{F}(x, t)=\int_{0}^{t} \tilde{f}(x, s) d s, \forall x \in \Omega, t \in R, \\
& \tilde{G}(x, t)=\int_{0}^{t} \tilde{g}(x, s) d s, \forall x \in \partial \Omega, t \in R, \\
& \tilde{b}(t)=\left\{\begin{array}{l}
b(t) \text { if } t \geq 0, \widehat{\tilde{b}}(t)=\int_{0}^{t} \tilde{b}(s) d s, \forall t \in R, \\
b(0) \text { if } t<0, \\
\tilde{E}(u)=\hat{a}\left(\int_{\Omega} \frac{1}{p(x)}\left(|\nabla u|^{p(x)}+|u|^{p(x)}\right) d x\right)-\widehat{\tilde{b}}\left(\int_{\Omega} \tilde{F}(x, u) \mathrm{d} x\right)-\int_{\partial \Omega} \tilde{G}(x, u) \mathrm{d} \sigma, \forall u \in X .
\end{array}\right.
\end{aligned}
$$

Then, using truncation functions above, similarly in the proof of Theorem 3.4 in [25], we can prove that $\widetilde{E}$ has a nontrivial global minimizer $u_{0}$ and $u_{0}$ is a nontrivial nonnegative solution of $(P)$.

\section{The Mountain Pass theorem}

In this section we will find the Mountain Pass type critical points of the energy functional $E$ associated with problem $(P)$.

Lemma 4.1. Let $\left(f_{1}\right),\left(g_{1}\right),\left(a_{1}\right),\left(b_{1}\right)$ and the following conditions hold true:

$\left(\mathrm{a}_{2}\right)^{\prime} \exists \alpha_{1}>0, M>0$, and $C>0$ such that

$\hat{a}(t) \geq C t^{\alpha_{1}}$ for all $t \geq M$

with $\alpha_{1} p_{-}>1$.

(a $\mathrm{a}_{4} \exists \lambda>0, M>0$ such that

$\lambda \hat{a}(t) \geq a(t) t$ for all $t \geq M$

(b $\left.\mathrm{b}_{4}\right) \exists \theta>0, M>0$ such that:

$0 \leq \theta \widehat{b}(t) \leq b(t) t$, for all $t \geq M$.

$\left(\mathrm{f}_{4}\right) \exists \mu>0, M>0$ such that:

$0 \leq \mu F(x, t) \leq f(x, t) t$, for $|t| \geq M$ and $x \in \Omega$.

$\left(\mathrm{g}_{4}\right) \exists \kappa>\theta \mu>0, M>0$ such that:

$0 \leq \kappa G(x, t) \leq g(x, t) t,|t| \geq M$ and $x \in \partial \Omega$.

$\left(\mathrm{H}_{3}\right) \lambda p_{+}<\theta \mu$.

Then E satisfies condition (P.S) $c$ for any $c \neq 0$.

Proof. By $\left(\mathrm{a}_{4}\right)$, for $\|u\|$ large enough,

$$
\begin{aligned}
\lambda p+J(u) & =\lambda p+\hat{a}\left(\int_{\Omega} \frac{1}{p(x)}\left(|\nabla u|^{p(x)}+|u|^{p(x)}\right) d x\right) \\
& \geq p+a\left(\int_{\Omega} \frac{1}{p(x)}\left(|\nabla u|^{p(x)}+|u|^{p(x)}\right) d x\right) \int_{\Omega} \frac{1}{p(x)}\left(|\nabla u|^{p(x)}+|u|^{p(x)}\right) d x \\
& \geq a\left(\int_{\Omega} \frac{1}{p(x)}\left(|\nabla u|^{p(x)}+|u|^{p(x)}\right) d x\right) \int_{\Omega}\left(|\nabla u|^{p(x)}+|u|^{p(x)}\right) d x=J^{\prime}(u) u .
\end{aligned}
$$


From $\left(\mathrm{f}_{4}\right)$ and $\left(\mathrm{g}_{4}\right)$ we can see that there exists $C_{1}>0$ and $C_{2}>0$ such that

$$
\begin{aligned}
& -C_{1} \leq \mu \int_{\Omega} F(x, u) d x \leq \int_{\Omega} f(x, u) u d x+C_{1}, \forall u \in X, \\
& -C_{2} \leq \kappa \int_{\partial \Omega} G(x, u) d \sigma \leq \int_{\partial \Omega} g(x, u) u d \sigma+C_{2}, \forall u \in X,
\end{aligned}
$$

and thus, given any $\varepsilon \in(0, \mu)$, there exists $M_{\varepsilon} \geq M>0$ and $M_{\varepsilon}^{\prime} \geq M>0$ such that

$$
\begin{gathered}
(\mu-\varepsilon) \int_{\Omega} F(x, u) d x \leq \int_{\Omega} f(x, u) u d x, \text { if } \int_{\Omega} F(x, u) d x \geq M_{\varepsilon,} \\
\theta(\mu-\varepsilon) \int_{\partial \Omega} G(x, u) d \sigma \leq \int_{\partial \Omega} g(x, u) u d \sigma, \text { if } \int_{\partial \Omega} G(x, u) d \sigma \geq M^{\prime}{ }_{\varepsilon} .
\end{gathered}
$$

We may assume $M_{\varepsilon}>\frac{c_{1}}{\mu}$ and $M_{\varepsilon}{ }^{\prime}>\frac{c_{2}}{\theta \mu}$. Note that in this case the inequalities $\int_{\Omega} F(x, u) d x \geq M_{\varepsilon}$ and $\int_{\partial \Omega} G(x, u) d \sigma \geq M_{\varepsilon}^{\prime}$ are equivalent to $\left|\int_{\Omega} F(x, u) d x\right| \geq M_{\varepsilon}$ and $\left|\int_{\partial \Omega} G(x, u) d \sigma\right| \geq M_{\varepsilon}^{\prime}$, because $\int_{\Omega} F(x, u) d x \geq-\frac{C_{1}}{\mu}$ and $\int_{\partial \Omega} G(x, u) d \sigma \geq-\frac{c_{2}}{\theta \mu}$ for all $u \in$ $X$. We claim that there exist $C_{\varepsilon}>0$ and $C_{\varepsilon}^{\prime}>0$ such that

$$
\begin{aligned}
& \Phi^{\prime}(u) u-\theta(\mu-\varepsilon) \Phi(u) \geq-C_{\varepsilon} \text { for } u \in X, \\
& \Psi^{\prime}(u) u-\theta(\mu-\varepsilon) \Psi(u) \geq-C_{\varepsilon}^{\prime} \text { for } u \in X .
\end{aligned}
$$

Indeed, when $\left|\int_{\Omega} F(x, u) d x\right| \leq M_{\varepsilon}$ and $\left|\int_{\partial \Omega} G(x, u) d \sigma\right| \leq M_{\varepsilon}^{\prime}$, the validity is obvious. When $\left|\int_{\Omega} F(x, u) d x\right| \geq M_{\varepsilon}$ and $\left|\int_{\partial \Omega} G(x, u) d \sigma\right| \geq M_{\varepsilon}^{\prime}$, i.e., $\int_{\Omega} F(x, u) d x \geq M_{\varepsilon}$ and $\int_{\partial \Omega} G(x, u) d \sigma \geq M_{\varepsilon}^{\prime}$, we have that

$$
\begin{aligned}
\theta(\mu-\varepsilon) \Phi(u) & =\theta(\mu-\varepsilon) \hat{b}\left(\int_{\Omega} F(x, u) \mathrm{d} x\right) \\
& \leq(\mu-\varepsilon) b\left(\int_{\Omega} F(x, u) \mathrm{d} x\right) \int_{\Omega} F(x, u) \mathrm{d} x \\
& \leq b\left(\int_{\Omega} F(x, u) \mathrm{d} x\right) \int_{\Omega} f(x, u) u \mathrm{~d} x=\Phi^{\prime}(u) u,
\end{aligned}
$$

and

$$
\begin{aligned}
\theta(\mu-\varepsilon) \Psi(u) & =\theta(\mu-\varepsilon) \int_{\partial \Omega} G(x, u) d \sigma \\
& \leq \int_{\partial \Omega} g(x, u) u d \sigma=\Psi^{\prime}(u) u .
\end{aligned}
$$

Now let $\left\{u_{n}\right\} \subset X \backslash\{0\}, E\left(u_{n}\right) \rightarrow c \neq 0$ and $E^{\prime}\left(u_{n}\right) \rightarrow 0$. By $\left(\mathrm{H}_{3}\right)$, there exists $\varepsilon>0$ small enough such that $\lambda p_{+}<\theta(\mu-\varepsilon)$. Then, since $\left\{u_{n}\right\}$ is a $(P . S)_{c}$ sequence, for sufficiently large $n$, we have

$$
\begin{aligned}
& \theta(\mu-\varepsilon) c+1+\left\|u_{n}\right\| \\
\geq & \theta(\mu-\varepsilon) E\left(u_{n}\right)-E^{\prime}\left(u_{n}\right) u_{n} \\
\geq \quad & \left(\theta(\mu-\varepsilon)-\lambda p_{+}\right) J\left(u_{n}\right)+\left(\lambda p_{+} J\left(u_{n}\right)-J^{\prime}\left(u_{n}\right) u_{n}\right)+\left(\Phi^{\prime}\left(u_{n}\right) u_{n}-\theta(\mu-\varepsilon) \Phi\left(u_{n}\right)\right) \\
& +\left(\Psi^{\prime}\left(u_{n}\right) u_{n}-\theta(\mu-\varepsilon) \Psi\left(u_{n}\right)\right) \\
\geq & C_{3}\left\|u_{n}\right\|^{\alpha_{1} p-}-C_{4}-C_{\varepsilon}-C_{\varepsilon}^{\prime}
\end{aligned}
$$

Since $\alpha_{1} p_{-}>1$, we have that $\left\{\left\|u_{n}\right\|\right\}$ is bounded. By Lemma 3.2, $E$ satisfies condition $(P . S)_{c}$ for $c \neq 0$. 
Theorem 4.1. Under the hypotheses of Lemma 4.1, and let the following conditions hold:

$\left(\mathrm{a}_{5}\right)$ There is a positive constant $\alpha_{3}$ such that $\limsup \frac{\hat{a}(t)}{t^{\alpha} 3}>0$.

$\left(\mathrm{b}_{5}\right)$ There is a positive constant $\beta_{3}$ such that $\liminf _{t \rightarrow 0} \frac{\hat{b}(t)}{|t|^{\beta_{3}}}<+\infty$.

$\left(\mathrm{f}_{5}\right)$ There exists $r_{1} \in C^{0}(\bar{\Omega})$ such that $1<r_{1}(x)<p^{* \prime}(x)$ for $x \in \bar{\Omega}$ and $\liminf _{t \rightarrow 0} \frac{|F(x, t)|}{|t|^{1}(x)}<+\infty$ uniformly for $x \in \Omega$.

$\left(\mathrm{g}_{5}\right)$ There exists such $r_{2} \in C^{0}(\bar{\Omega})$ such that $1<r_{2}(x)<p^{*}(x)$ for $x \in \partial \Omega$ and $\liminf _{t \rightarrow 0} \frac{|G(x, t)|}{|t|^{r_{2}}(x)}<+\infty$ uniformly for $x \in \partial \Omega$.

$\left(\mathrm{H}_{4}\right) \alpha_{3} p_{+}<\beta_{3} r_{1-}, \alpha_{3} p_{+}<r_{2-}, \lambda p_{+}<\theta \mu$.

Then $(P)$ has a nontrivial solution with positive energy.

Proof. Let us prove this conclusion by the Mountain Pass lemma. $E$ satisfies condition $(P . S)_{c}$ for $c \neq 0$ has been proved in Lemma 4.1.

For $\|u\|$ small enough, from $\left(a_{5}\right)$ we can obtain easily that $J(u) \geq C_{1}\|u\|^{\alpha_{3} p_{+}}$, from $\left(b_{5}\right),\left(f_{1}\right)$ and $\left(f_{5}\right)$ we have $|\Phi(u)| \leq C_{2}\|u\|^{\beta_{3} r_{1-}}$, and in the similar way from $\left(g_{1}\right)$ and $\left(g_{5}\right)$ we have $|\Psi(u)| \leq C_{2}\|u\|^{r_{2}-}$. Thus by $\left(\mathrm{H}_{4}\right)$, we conclude that there exist positive constants $\rho$ and $\delta$ such that $E(u) \geq$ for $\|u\|=\rho$.

Let $w \in X \backslash\{0\}$ be given. From $\left(\mathrm{a}_{4}\right)$ for sufficiently large $t>0$ we have $\hat{a}(t) \leq C_{1} t^{\lambda}$, which follows that $J(s w) \leq d_{1} s^{\lambda p_{+}}$for $s$ large enough, where $d_{1}$ is a positive constant depending on $w$. From $\left(f_{4}\right)$ and $\left(f_{1}\right)$ for $|t|$ large enough we have $\int_{\Omega} F(x, s w) \mathrm{d} x \geq d_{2} s^{\mu}$ for $s$ large enough, where $d_{2}$ is a positive constant depending on $w$. From $\left(\mathrm{b}_{4}\right)$ for $t$ large enough we have $\Phi(s w)=\hat{b}\left(\int_{\Omega} F(x, s w) \mathrm{d} x\right) \geq d_{3} s^{\theta \mu}$ for $s$ large enough, where $d_{3}$ is a positive constant depending on $w$. From $\left(\mathrm{g}_{4}\right)$ and $\left(\mathrm{g}_{1}\right)$ for $|t|$ large enough we have $\Psi(s w)=\int_{\partial \Omega} G(x, s w) d \sigma \geq d_{4} s^{\theta \mu}$. Hence for any $w \in X \backslash\{0\}$ and $s$ large enough, $E(s w) \leq d_{1} s^{\lambda p_{+}}-d_{3} s^{\theta \mu}-d_{4} s^{\theta \mu}$, thus by $\left(\mathrm{H}_{3}\right)$, We conclude that $E(s w) \rightarrow-\infty$ as $s \rightarrow$ $+\infty$.

So by the Mountain Pass lemma this theorem is proved.

By the symmetric Mountain Pass lemma, similarly in the proof of Theorem 4.8 in [40], we have the following:

Theorem 4.2. Under the hypotheses of Theorem 4.1, if, in addition, $\left(\mathrm{f}_{3}\right)$ and $\left(\mathrm{g}_{3}\right)$ are satisfied, then $(P)$ has a sequence of solutions $\left\{ \pm u_{n}\right\}$ such that $E\left( \pm u_{n}\right) \rightarrow+\infty$ as $n \rightarrow \infty$.

\section{Acknowledgements}

The authors thank the two referees for their careful reading and helpful comments of the study. Research supported by the National Natural Science Foundation of China (10971088), (10971087).

\section{Authors' contributions}

EG and PZ contributed to each part of this work equally. All the authors read and approved the final manuscript.

\section{Competing interests}

The authors declare that they have no competing interests.

Received: 29 August 2011 Accepted: 4 January 2012 Published: 4 January 2012

References

1. Kirchhoff, G: Mechanik. Teubner, Leipzig (1883)

2. Lions, JL: On some questions in boundary value problems of mathematical physics. In: Rio de Janeiro 1977, in: de la Penha, Medeiros (eds.) Proceedings of International Symposium on Continuum Mechanics and Partial Differential Equations Math Stud North-Holland. 30, 284-346 (1978) 
3. Arosio, A, Panizzi, S: On the well-posedness of the Kirchhoff string. Trans Am Math Soc. 348, 305-330 (1996). doi:10.1090/50002-9947-96-01532-2

4. Cavalcanti, MM, Domingos Cavalcanti, VN, Soriano, JA: Global existence and uniform decay rates for the KirchhoffCarrier equation with nonlinear dissipation. Adv Diff Equ. 6, 701-730 (2001)

5. Chipot, M, Lovat, B: Some remarks on non local elliptic and parabolic problems. Nonlinear Anal. 30, 4619-4627 (1997). doi:10.1016/S0362-546X(97)00169-7

6. D'Ancona, P, Spagnolo, S: Global solvability for the degenerate Kirchhoff equation with real analytic date. Invent Math. 108, 447-462 (1992)

7. Chipot, M, Rodrigues, JF: On a class of nonlocal nonlinear elliptic problems. RAIRO Modélisation Math Anal Numbér. 26 , 447-467 (1992)

8. Dreher, M: The Kirchhoff equation for the p-Laplacian. Rend Semin Mat Univ Politec Torino. 64, 217-238 (2006)

9. Dreher, M: The wave equation for the p-Laplacian. Hokkaido Math J. 36, 21-52 (2007)

10. Autuori, G, Pucci, P, Salvatori, MC: Asymptotic stability for anistropic Kirchhoff systems. J Math Anal Appl. 352, 149-165 (2009). doi:10.1016/j.jmaa.2008.04.066

11. Perera, K, Zhang, ZT: Nontrivial solutions of Kirchhoff-type problems via the Yang index. J Diff Equ. 221, 246-255 (2006). doi:10.1016/j.jde.2005.03.006

12. Alves, CO, Corrêa, FJSA, Ma, TF: Positive solutions for a quasilinear elliptic equation of Kirchhoff type. Comput Math Appl. 49, 85-93 (2005). doi:10.1016/j.camwa.2005.01.008

13. Corrêa, FJSA, Figueiredo, GM: On an elliptic equation of p-Kirchhoff type via variational methods. Bull Aust Math Soc. 74, 263-277 (2006). doi:10.1017/S000497270003570X

14. Corrêa, FJSA, Figueiredo, GM: On a p-Kirchhoff equation via Krasnosel-skii's genus. Appl Math Lett. 22, 819-822 (2009). doi:10.1016/j.aml.2008.06.042

15. Corrêa, FJSA, Menezes, SDB, Ferreira, J: On a class of problems involving a nonlocal operator. Appl Math Comput. 147 475-489 (2004). doi:10.1016/50096-3003(02)00740-3

16. He, XM, Zou, WM: Infinitly many positive solutions for Kirchhoff-type problems. Nonlinear Anal. 70, 1407-1414 (2009). doi:10.1016/j.na.2008.02.021

17. Fan, XL: On the sub-supersolution method for $p(x)$-Laplacian equations. J Math Anal Appl. 330, 665-682 (2007). doi:10.1016/j.jmaa.2006.07.093

18. Fan, $\mathrm{XL}, \mathrm{Han}, \mathrm{XY}$ : Existence and multiplicity of solutions for $p(x)$-Laplacian equations in $R^{n}$. Nonlinear Anal. 59, 173-188 (2004)

19. Fan, XL, Shen, JS, Zhao, D: Sobolev embedding theorems for space $W^{k, p(x)}(\Omega)$. J Math Anal Appl. 262, 749-760 (2001). doi:10.1006/jmaa.2001.7618

20. Fan, XL, Zhang, QH: Existence of solutions for $p(x)$-Laplacian Dirichlet problems. Nonlinear Anal. 52, 1843-1852 (2003). doi:10.1016/50362-546X(02)00150-5

21. Fan, XL, Zhang, QH, Zhao, D: Eigenvalues of $p(x)$-Laplacian Dirichlet problem. J Math Anal Appl. 302, 306-317 (2005). doi:10.1016/j.jmaa.2003.11.020

22. Fan, $X L$, Zhao, D: On the spaces $L^{p(x)}(\Omega)$ and $W^{k, p(x)}(\Omega)$. J Math Anal Appl. 263, 424-446 (2001). doi:10.1006/ jmaa.2000.7617

23. Fan, $\mathrm{XL}$, Zhao, $\mathrm{YZ}$, Zhang, QH: A strong maximum principle for $p(x)$-Laplacian equations. Chinese Ann Math Ser A 24, 495-500 (2003). (in Chinese); Chinese J Contemp Math 24: 277-282 (2003)

24. Dai, GW, Hao, RF: Existence of solutions for a p(x)-Kirchhoff-type equation. J Math Anal Appl. 359, 275-284 (2009). doi:10.1016/j.jmaa.2009.05.031

25. Fan, XL: On nonlocal $p(x)$-Laplacian Dirichlet problems. Nonlinear Anal. 72, 3314-3323 (2010). doi:10.1016/j. na.2009.12.012

26. Chipot, M, Shafrir, I, Fila, M: On the solutions to some elliptic equations with nonlinear boundary conditions. Adv Diff Eq. 1, 91-110 (1996)

27. Hu, B: Nonexistence of a positive solution of the Laplace equation with a nonlinear boundary condition. Diff Integral Equ. 7(2), 301-313 (1994)

28. dal Maso, Gianni, Ebobisse, Francois, Ponsiglione, Marcello: A stability result for nonlinear Neumann problems under boundary variations. J Math Pures Appl. 82, 503-532 (2003). doi:10.1016/S0021-7824(03)00014-X

29. Garcia-Azorero, J, Peral, I, Rossi, JD: A convex-concave problem with a nonlinear boundary condition. J Diff Equ. 198, 91-128 (2004). doi:10.1016/S0022-0396(03)00068-8

30. Song, XC, Wang, WH, Zhao, PH: Positive solutions of elliptic equations with nonlinear boundary conditions. Nonlinear Anal. 70, 328-334 (2009). doi:10.1016/..na.2007.12.003

31. Bonder, JF, Pinasco, JP, Rossi, JD: Existence results for Hamiltonian elliptic systems with nonlinear boundary conditions. Electron J Diff Equ. 40, 1-15 (1999)

32. Bonder, JF, Rossi, JD: Existence for an elliptic system with nonlinear boundary conditions via fixed point methods. Adv Diff Equ. 6, 1-20 (2001)

33. Bonder, JF, Rossi, JD: Existence results for the $p$-Laplacian with nonlinear boundary conditions. J Math Anal Appl. 263, 195-223 (2001). doi:10.1006/jmaa.2001.7609

34. Cîrstea, STFlorica-Corina, Rădulescu, DVicentiu: Existence and nonexistence results for a quasilinear problem with nonlinear boundary condition. J Math Anal Appl. 244, 169-183 (2000). doi:10.1006/jmaa.1999.6699

35. Afrouzi, GA, Alizadeh, M: A quasilinearization method for $p$-Laplacian equations with a nonlinear boundary condition. Nonlinear Anal. 71, 2829-2833 (2009). doi:10.1016/j.na.2009.01.134

36. Martinez, S, Rossi, JD: On the Fučik spectrum and a resonance problem for the $p$-Laplacian with a nonlinear boundary condition. Nonlinear Anal. 59, 813-848 (2004)

37. Afrouzi, GA, Rasouli, SH: A variational approach to a quasilinear elliptic problem involving the $p$-Laplacian and nonlinear boundary condition. Nonlinear Anal. 71, 2447-2455 (2009). doi:10.1016/j.na.2009.01.090

38. Deng, SG, Wang, Q: Nonexistence, existence and multiplicity of positive solutions to the $p(x)$-Laplacian nonlinear Neumann boundary value problem. Nonlinear Anal. 73, 2170-2183 (2010). doi:10.1016/..na.2010.05.043

39. Deng, SG: A local mountain pass theorem and applications to a double perturbed $p(x)$-Laplacian equations. Appl Math Comput. 211, 234-241 (2009). doi:10.1016/j.amc.2009.01.042 
40. Yao, JH: Solutions for Neumann boundary value problems involving $p(x)$-Laplace operators. Nonlinear Anal. 68, 1271-1283 (2008). doi:10.1016/j.na.2006.12.020

41. Edmunds, DE, Rákosník, J: Density of smooth functions in $W^{k, p(x)}(\Omega)$. Proc R Soc A. 437, $229-236$ (1992). doi:10.1098/ rspa.1992.0059

42. Edmunds, DE, Rákosník, J: Sobolev embedding with variable exponent. Studia Math. 143, 267-293 (2000)

doi:10.1186/1687-2770-2012-1

Cite this article as: Guo and Zhao: Existence and multiplicity of solutions for nonlocal $p(x)$-Laplacian equations with nonlinear Neumann boundary conditions. Boundary Value Problems 2012 2012:1.

\section{Submit your manuscript to a SpringerOpen ${ }^{\circ}$} journal and benefit from:

- Convenient online submission

- Rigorous peer review

- Immediate publication on acceptance

- Open access: articles freely available online

- High visibility within the field

- Retaining the copyright to your article

Submit your next manuscript at $\boldsymbol{s p r i n g e r o p e n . c o m ~}$ 